Mots. Les langages du politique

\title{
Le traitement de la crise économique par les agences de presse : une comparaison France/ Espagne
}

The processing of the economic crisis by the news agencies: a comparison

France/Spain

El tratamiento de la crisis económica por agencias de noticias : una comparación entre Francia/España

Mariola Moreno, Pierre Ratinaud et Pascal Marchand

\section{OpenEdition}

Journals

Édition électronique

URL : http://journals.openedition.org/mots/22927

DOI : $10.4000 /$ mots.22927

ISSN : 1960-6001

Éditeur

ENS Éditions

Édition imprimée

Date de publication : 1 novembre 2017

Pagination : $51-72$

ISSN : 0243-6450

Référence électronique

Mariola Moreno, Pierre Ratinaud et Pascal Marchand, «Le traitement de la crise économique par les agences de presse : une comparaison France/Espagne », Mots. Les langages du politique [En ligne], 115 | 2017, mis en ligne le 21 novembre 2019, consulté le 05 janvier 2021. URL : http:// journals.openedition.org/mots/22927 ; DOI : https://doi.org/10.4000/mots.22927 


\section{Le traitement de la crise économique par les agences de presse : une comparaison France/Espagne*}

La crise économique possède une «réalité », constituée de ses impacts observables, de ses conséquences pour les opérateurs, les mouvements des marchés financiers, la vie quotidienne des personnes et des organisations. Mais elle constitue également un objet de traitement par les médias et la communication politique, en soi (ses effets, causes et conséquences) ou comme référence invoquée pour le traitement d'autres objets (économiques, politiques, sociaux, etc.), ce qui justifie l'intérêt porté aux dynamiques psychosociales (De Rosa, Bocci et Bulgarella, 2010).

Pour devenir un tel objet de diffusion et émerger dans la sphère publique, l'objet «crise » doit subir des opérations de simplification et de catégorisation pour le doter d'un sens commun. Serge Moscovici (1961, p. 506) invoquait les processus d'objectivation (description, explication, schématisation, etc.) et d'ancrage (analogies, comparaisons, mises en relation, etc.). Nous poserons l'hypothèse que l'organisation lexicale des articles de presse traitant de la crise est à même de rendre compte de ces effets d'ancrage. Les principales agences de presse en France, l'Agence France-Presse (AFP), et en Espagne (EFE) ${ }^{1}$, doivent nous permettre d'appréhender de tels ancrages, communs aux deux pays ou spécifiques à l'un d'eux, pour comprendre la genèse et la diffusion des représentations sociales de la crise économique. L'utilisation du logiciel Iramuteq (Ratinaud, 2014) et notamment de la méthode Reinert (Reinert, 1983 et 1990 ; Ratinaud et Marchand, 2012), permettra de faire

* Ce travail a été réalisé dans le cadre du LABEX SMS portant la référence ANR-11-LABX-0066

1. Il y a plusieurs hypothèses par rapport à la signification de l'acronyme de l'agence EFE : cela pourrait correspondre à la lettre $\mathrm{F}$ du parti phalangiste (Falange en espagnol), à un hommage aux initiales du dictateur (Francisco Franco) ou encore faire référence aux trois initiales des trois agences (Fabra, Febus, Faro) qui existaient en Espagne avant l'arrivée de l'EFE.

Université Toulouse 3-Paul-Sabatier, LERASS

mariolamorenocalvo@gmail.com

Université Toulouse 2-Jean-Jaurès, LERASS

ratinaud@univ-tlse2.fr

Université Toulouse 3-Paul-Sabatier, LERASS

pascal.marchand@univ-tlse3.fr 
apparaître les similitudes et les différences des thématiques abordées dans les deux contextes distincts (France et Espagne) à la fois dans leur contenu et dans la temporalité de leur expression.

\section{La crise et les représentations sociales}

Pour S. Moscovici (1961, p. 506), la création d'une représentation sociale s'observe au travers de processus historiques ou empiriques complexes, qui ne se limitent pas à la pensée institutionnelle, mais impliquent l'influence des expériences et des échanges communicationnels quotidiens entre les individus et dans un contexte particulier.

Ainsi, les représentations sociales ne sont pas égales dans toutes les sociétés : si, d'après l'hypothèse des Nexus de Sébastien Rouquette (1988, p. 127 et 1994, p. 221), certaines peuvent avoir un caractère universel dans certaines circonstances («nazi », « liberté », « justice», etc.), les autres sont particulières aux groupes sociaux qui les ont produites. Dans la genèse et la diffusion des représentations sociales dans un groupe social, le rôle que jouent les médias, comme générateurs de prises de position (Bourdieu, 1972, p. 272), doit être particulièrement considéré.

Denise Jodelet (2011) a proposé certaines hypothèses sur le rôle des médias face à la crise financière mondiale, qui mobilisent l'ancrage : «Les médias, prenant parfois le relais des milieux scientifiques, donnent des situations de vie et de l'état du monde social, politique et économique des interprétations qui interviennent dans la formation et la formulation des peurs sociales». La crise économique, au-delà d'un certain volume d'information médiatique, devient non seulement une référence explicative d'autres événements, mais également un vecteur de positionnement à leur égard (Rizzoli, Romaioli et Contarello, 2017).

Mais, de l'objet initial à sa représentation telle qu'elle est diffusée par la presse, une étape intermédiaire nous intéresse particulièrement et concerne le travail des agences de presse. Un positionnement plus factuel constitue la base même de la production agencière (Palmer, 2009). Les questions de fiabilité et d'objectivité vont être deux piliers essentiels pour les journalistes des agences de presse, qui sont chargés de fournir des informations pour d'autres médias (télévisions, radios et journaux). Et pour parvenir à tous les médias - quelle que soit leur ligne éditoriale - ces informations doivent se présenter comme neutres et impartiales. La loi de 1957 sur le statut de l'AFP impose effectivement aux journalistes de l'agence de fournir une information «complète, objective, exacte, impartiale et digne de confiance» (Lagneau, 2003).

On considère donc que les agences de presse sont moins marquées que le reste des médias par l'éditorialisation, que l'on peut définir ici comme l'inscription de l'information dans un univers axiologique, marqué par un contrat 
de lectorat particulier (Burguet, 2000 ; Verón, 1985, p. 527). Il s’agit donc, pour en rester à la perspective de S. Moscovici (1961, p. 506), de privilégier dans ce travail une presse de diffusion, par opposition à une presse de propagation ou de propagande.

Si l'on accepte ce postulat, le traitement de l'information par les agences de presse est à même de révéler la relation existant entre une réalité sociohistorique (la «crise») et les bases de construction des représentations sociales qui se diffuseront dans les organes médiatiques.

Il ne s'agit pas d'affirmer que ce traitement par les agences de presse est plus « objectif» que celui des rédactions postérieures par les organes de presse qui peuvent s'en inspirer. On posera au contraire l'hypothèse qu'il traduit une certaine façon de vivre l'évènement, en puisant dans les contextes généraux qui fournissent des éléments d'ancrage (Doise, 1982, p. 233) et impliquent la culture, l'importance de l'objet, les caractéristiques de l'identité nationale (Marchand et Ratinaud, 2012a, p. 318). Ce traitement peut également refléter des routines propres aux représentations professionnelles des journalistes (Bataille, Blin, Jacquet-Mias et Piaser, 1997) qui impliquent des aspects globaux et des aspects locaux. Au plan global, on retiendra des formalisations mondialisées de l'information médiatique, une lecture homogène de l'information, des schémas d'écriture transversaux (par exemple, le modèle de la pyramide inversée : Castéran, 2012, p. 18). Au plan local, les spécificités culturelles et nationales introduiront des différences et des ressemblances dans les traitements de la crise par les agences de différents pays.

\section{Corpus et méthode}

On se donnera pour objectif de saisir la dynamique de construction des représentations sociales et, notamment, les modes d'ancrage qui ont présidé à leur formation, en comparant le traitement médiatique dans deux pays, la France et l'Espagne, frappés par la même crise mondiale mais dans des contextes économiques, sociaux et culturels distincts, et avec des conséquences différentes. Ces caractéristiques sont reflétées dans les dépêches de chaque agence.

Le choix de travailler sur des dépêches d'agences de presse permet également de constituer des corpus relevant du même genre, facilitant la comparaison. Ces corpus ont été constitués sur la base des mots-clés «crise économique/crisis económica». La question se pose de limiter la recherche aux dépêches contenant la locution «crise économique » plutôt que la forme simple «crise». Cette décision a été prise après avoir observé la polysémie du mot "crise », particulièrement dans la presse française, que ce soit pour qualifier d'autres contextes ("crise sociale », «crise de régime », etc.), ou dans le cadre d'emplois plus métaphoriques («crise de nerfs», etc.). 
Les dépêches des agences française (AFP) et espagnole (EFE) 2 ont donc été extraites de la base de données de presse Factiva dans le cas de l'agence espagnole et sur Europresse dans le cas français, de janvier 2008 à mars 2014. Le logiciel libre Iramuteq (Ratinaud, 2014) d'analyse textométrique a été utilisé pour rendre compte des structures lexicales et des thématiques abordées dans les deux corpus.

Ces analyses utiliseront des segments de texte comme unité d'analyse : les corpus sont découpés en segments d'une quarantaine d'occurrences en tenant compte de la ponctuation. Dans ces segments, une distinction est faite entre les formes «pleines » (les substantifs, les adjectifs, les verbes et les adverbes) et les mots outils (les pronoms, les conjonctions, les auxiliaires, etc.). Seules les formes pleines sont analysées.

Les caractéristiques des corpus obtenus sont décrites dans le tableau 1 :

Tableau 1 : description des corpus de l'AFP et de L'EFE

\begin{tabular}{lccc}
\hline & textes & occurrences & formes \\
\hline AFP & 28643 & 14279109 & 85738 \\
\hline EFE & 38147 & 16528190 & 100473 \\
\hline
\end{tabular}

La légère différence de taille entre les deux corpus peut être due à un effet de contexte (différence d'importance de la question de la crise économique dans chacun des deux pays) ou d'agenda médiatique (différence d'importance du traitement de cette question par les agences de presse) ou encore à l'utilisation ou pas de l'expression «crise économique» pour parler de la «crise». La taille moyenne des dépêches est comparable pour les deux agences (le nombre moyen d'occurrences par dépêche est de 498 pour l'AFP et de 433 pour l'EFE).

La comparaison des analyses menées sur ces deux corpus comprend deux étapes : une première analyse, que nous appelons macrostructurale, décrit les relations entre les mots les plus fréquents; une deuxième analyse, que nous appelons microstructurale, repose sur la classification de segments de textes.

\section{Une ressemblance macrostructurale}

Nous commencerons par la présentation d'analyses de similitude portant sur chacun des corpus. Il s'agit d'une représentation graphique des relations de cooccurrences entre les formes dans les segments de texte. Le point de départ de cette analyse est une matrice de similitude construite sur les formes pleines du corpus. Cette matrice compte, pour chaque couple de mots, le nombre de

2. Les sigles AFP et EFE seront utilisés pour renvoyer aux deux corpus d'étude. 
cooccurrences dans les segments, c'est-à-dire le nombre de segments contenant simultanément les deux mots. Cette matrice peut être représentée sous la forme d'un graphe de similitude. Dans ce graphe, les mots deviennent des nœuds et les cooccurrences de mots sont représentées par un lien entre ces nœuds. Les graphes bruts contiennent trop de liens pour être visualisés. Nous utilisons donc l'arbre maximum pour simplifier ce graphe. Il est obtenu en supprimant le lien le plus faible de chaque clique de trois nœuds. L'arbre maximum est donc le graphe connexe et sans cycle le plus «lourd» (en terme d'information) et il donne à lire les relations lexicales les plus fortes entre les formes les plus fréquentes d'un corpus.

L'arbre maximum pour le corpus AFP a été construit avec les 110 mots de fréquence supérieure à 4,755:

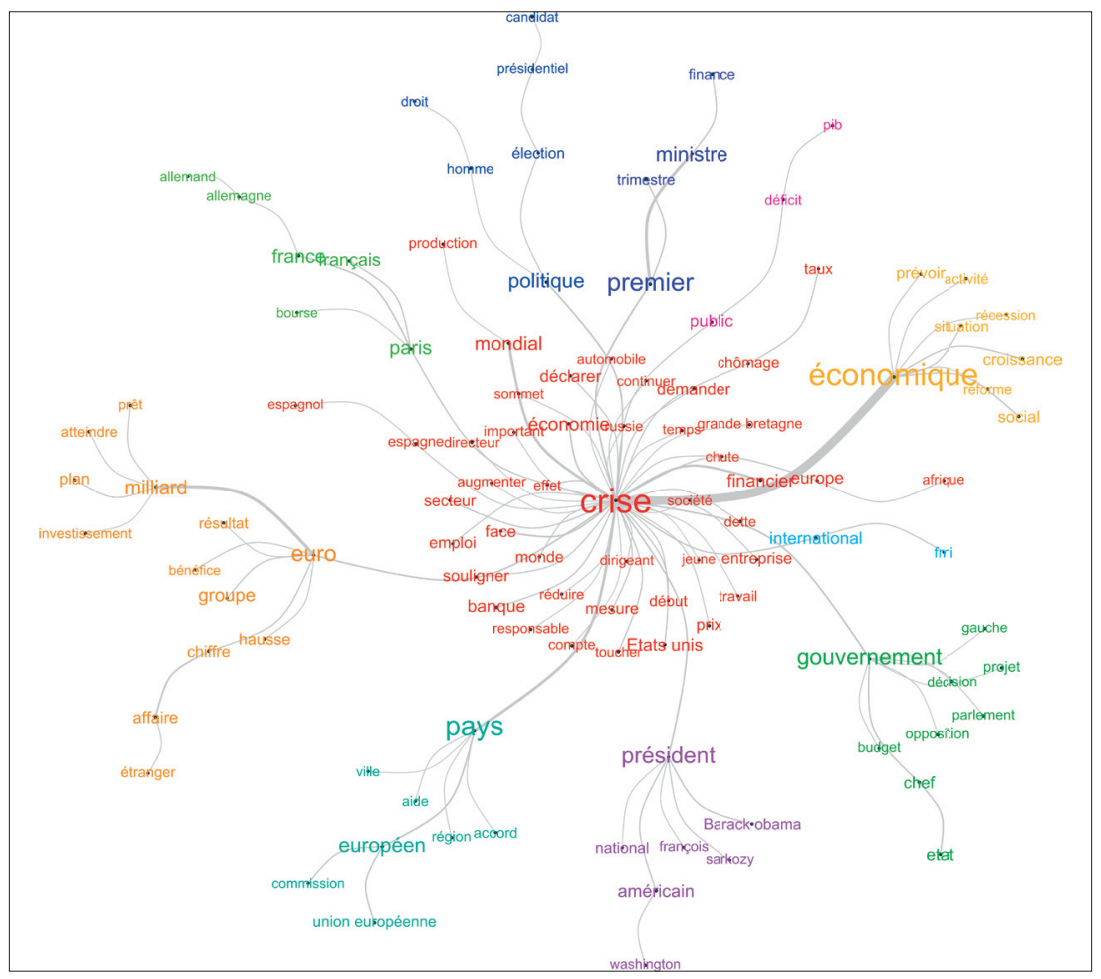

Figure 1 : Arbre maximum de l'analyse de similitude en cooccurrence sur les 110 formes pleines les plus fréquentes du corpus de l'AFP 
L'arbre maximum pour le corpus EFE a été construit avec les 110 mots de fréquence supérieure à 4,814 :

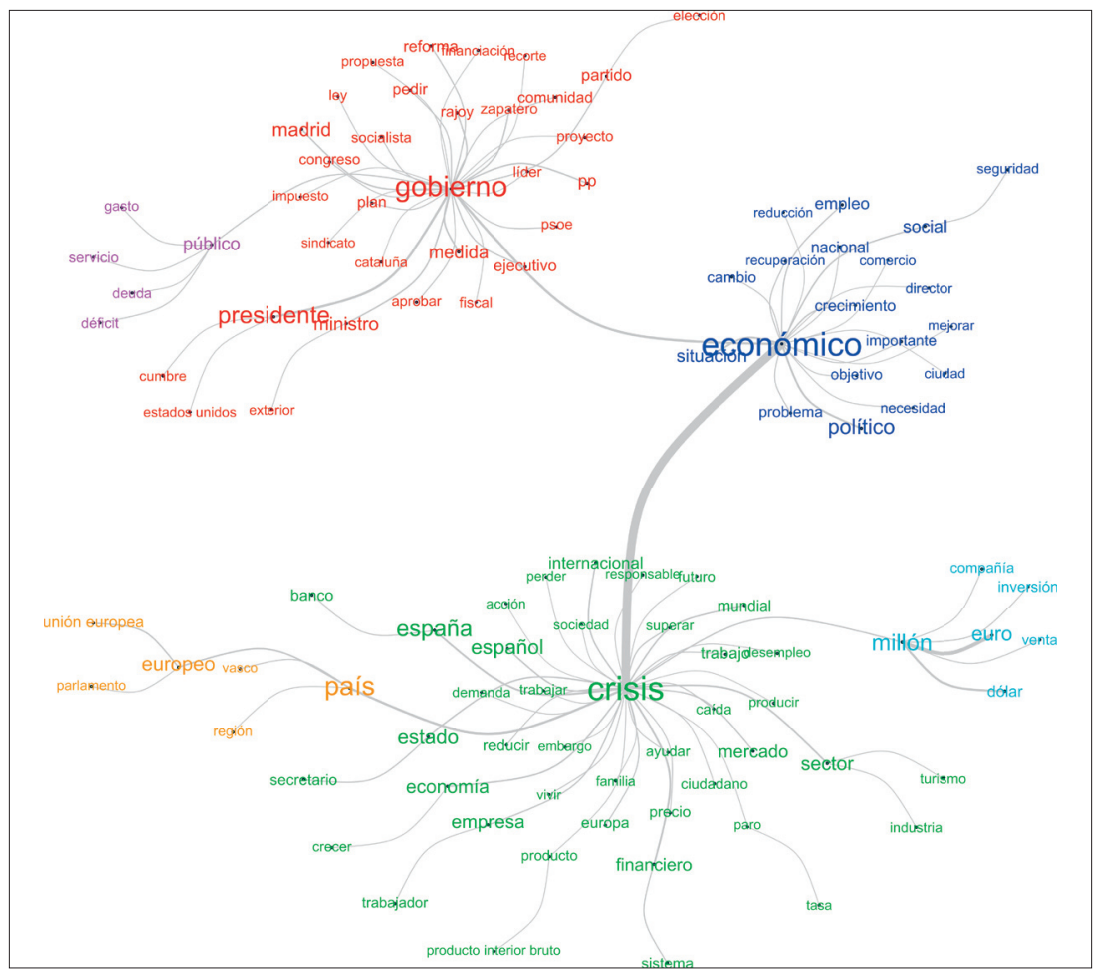

Figure 2 : Arbre maximum de l'analyse de similitude en cooccurrence des 110 formes pleines les plus fréquentes du corpus de l’EFE

Ces graphes de similitude nous donnent essentiellement à voir les convergences entre les deux corpus qui révèlent des distributions lexicales très proches. De façon prévisible, les deux graphes s'organisent autour des mots-clés «crise» et «économique» qui structurent le corpus. Mais le lemme «économique» centralise un champ lexical autour de notions plus générales comme social/social, situation/situación, croissance/crecimiento. Et le mot «crise» inclut autour de lui un lexique autour des effets et conséquences qui suppose une récession économique dans un pays, économie/economía, secteur/sector, monde/mundial.

Nous retrouvons également des champs lexicaux définis par les relations entre des formes de haute fréquence qui sont très convergentes :

- Un champ fait référence au pays/país autour duquel se mettent en relation des considérations nationales et internationales avec un lien fort avec l’Europe/europeo; 
- Un autre champ traite de la politique gouvernementale (gouvernement/ gobierno, président/presidente, ministre/ministro) mais, dans le cas espagnol, gouvernement et président sont liés, ce qui n'est pas le cas de l'AFP.

- Un champ mobilise des chiffres (euro/euro, dollar/dólar, milliard/millón) renforçant la nature économique et monétaire de la crise.

Nous proposons de parler de macrostructure pour rendre compte de cette organisation des termes les plus fréquents, qui révèle, peu ou prou, les mêmes champs lexicaux, validant l'hypothèse de routines communes et d'un traitement mondialisé de l'information médiatique. Si l'analyse s'arrêtait ici, une convergence dans la lecture des événements liés à la crise pourrait être évoquée.

Il faut néanmoins envisager que ces traitements, bien qu'ils soient instructifs, sont limités aux formes les plus fréquentes et aux relations de plus forte similitude entre ces formes. Nous préférerons donc les utiliser davantage pour valider la constitution des deux corpus et la pertinence de leur comparaison : ils parlent bien de la même chose et globalement dans les mêmes termes, mais l'analyse comparative ne peut en rester là.

\section{Des différences microstructurales}

Nous avons soumis les deux corpus à une classification lexicale permettant, au-delà des termes de haute fréquence, de mettre en évidence les thématiques (Ratinaud et Marchand, 2015) telles qu'elles sont abordées par chacune des agences, ainsi que leur organisation.

Nous utilisons une méthode proposée par M. Reinert (1983) et intégrée au logiciel Iramuteq. L'algorithme de Classification descendante hiérarchique (CDH) permet de définir des classes lexicales en partant du corpus global pour descendre progressivement vers les classes «terminales». L'analyse part d'une matrice de présence/absence qui croise les formes pleines retenues et les segments de texte du corpus. Cette matrice va subir une série de bipartitions (coupure de la matrice en deux) par l'intermédiaire d'une analyse factorielle des correspondances. À chaque étape, l'analyse regroupe les segments qui contiennent les mêmes formes. Les classes terminales obtenues sont des ensembles de segments de texte qui ont tendance à partager le même lexique. Il est alors possible de décrire ces classes à partir de leur profil lexical, c'està-dire à partir des mots qui sont statistiquement surreprésentés dans les seg. ments qui composent les classes.

La CDH permet d'identifier neuf classes pour l'AFP (95,96\% de segments classés) et neuf aussi pour l'EFE (99,95\% de segments classés). Les graphiques suivants sont les dendrogrammes des classifications auxquels on ajoute le vocabulaire statistiquement surreprésenté dans chacune des classes (par $\chi^{2}$ décroissant). 


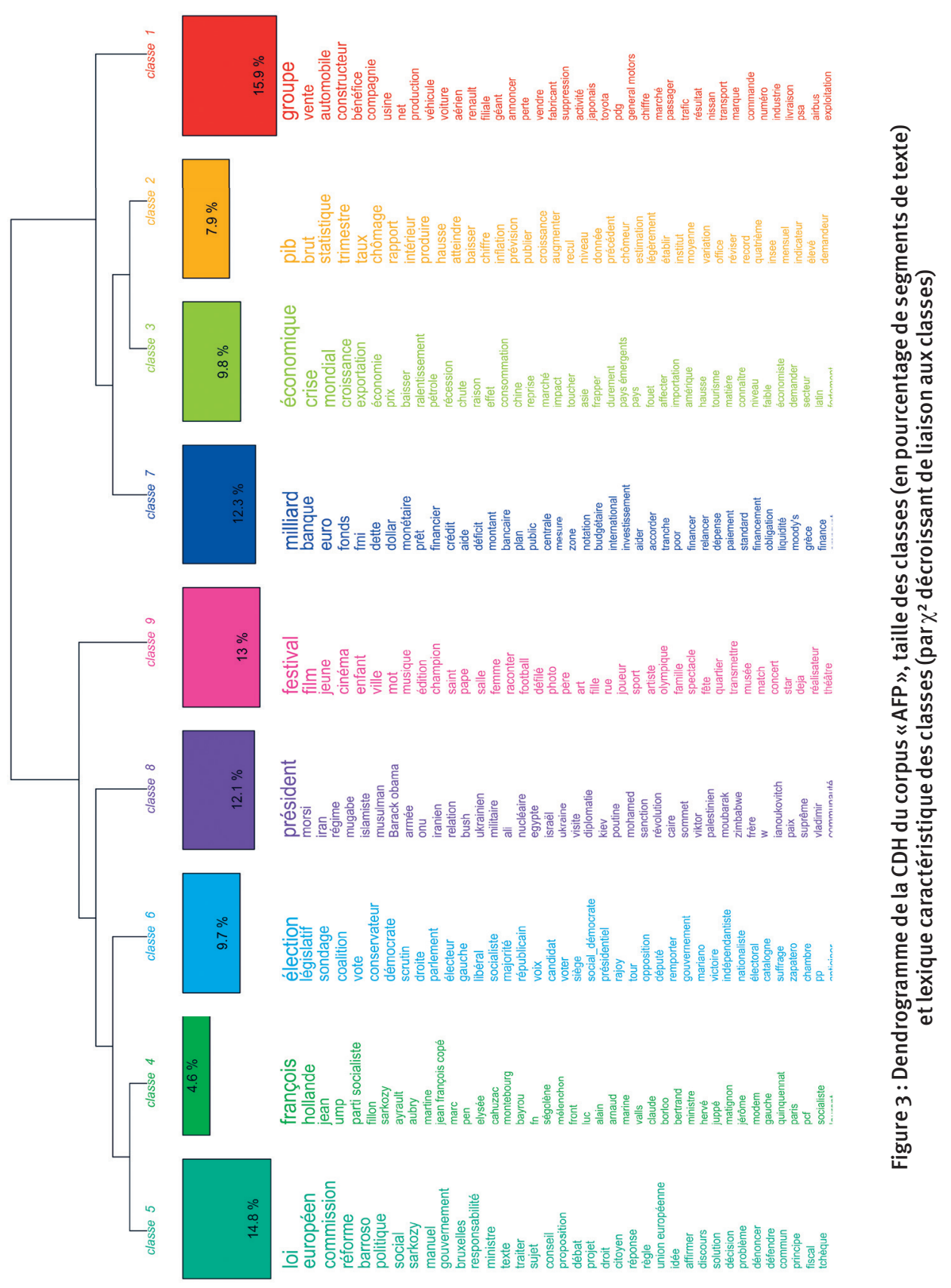




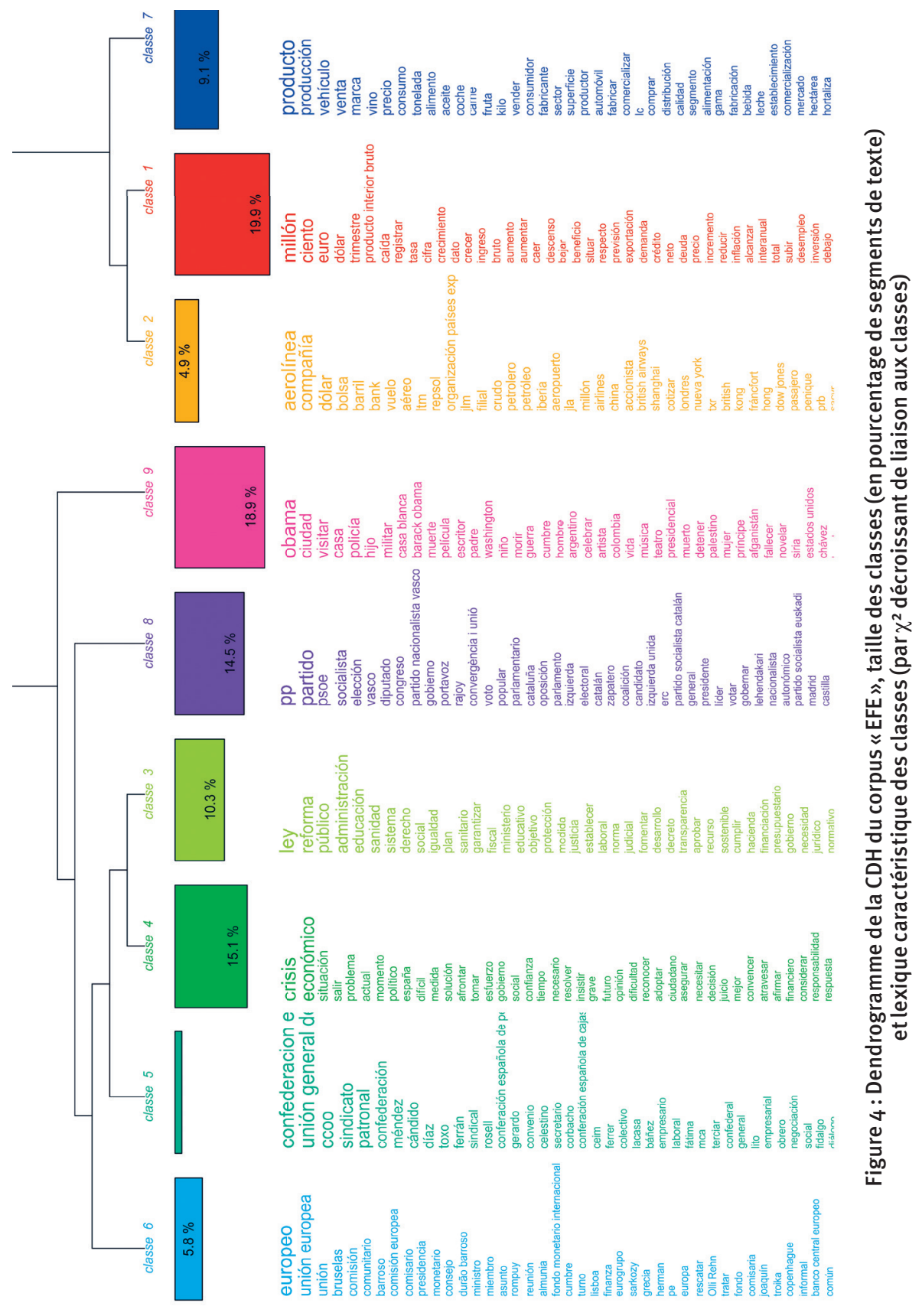


Nous retrouvons, dans ces deux classifications, les champs lexicaux identifiés par l'analyse de similitude et les convergences pointées précédemment dans les deux macrostructures. Ces deux CDH font apparaître une opposition similaire entre des classes traitant les considérations économiques et celles qui portent sur la politique.

Le corpus AFP oppose ainsi les classes 1, 2, 3 et 7, qui manifestent un vocabulaire économique, aux classes 4, 5, 6 et 8 qui portent sur la politique nationale et internationale. Le corpus de l'EFE oppose également les considérations économiques (classes 1, 2 et 7) aux considérations politiques (classes 3, 4, 5, 6, 8 et 9).

Le lexique économique fait référence aux questions monétaires, aux indicateurs qui montrent la situation de crise, ainsi qu'à l'activité économique en général et aux secteurs particulièrement touchés par la crise.

Le lexique politique de l'AFP se subdivise, d'une part en considérations électorales, législatives et internationales, et d'autre part en mesures et en lois mises en place par l'Europe pour sortir de la crise.

François Hollande qui avait lancé sa campagne fin janvier en estimant que son adversaire était le monde de la finance a ainsi ajouté un étage supplémentaire à la réforme fiscale qu'il entend appliquer s'il est élu à la présidence française lors des scrutins des 22 avril et 6 mai prochains. (27/02/2012, classe 4 de l'AFP)

Dans le corpus de l'EFE les questions politiques portent sur les politiques nationales et internationales, les mesures d'austérité, les manifestations syndicales et le manque de confiance sociale.

El vicesecretario general de organización del PP Carlos Floriano ha negado hoy que exista desmoralización en el partido ante las perspectivas económicas y de desempleo y se ha mostrado convencido de que las únicas políticas posibles para hacer frente a la crisis son las que ha puesto en marcha el gobierno.3 (29/04/2013, classe 4 de l'EFE)

Une première lecture des deux $\mathrm{CDH}$ confirme donc les convergences des deux structures de lexiques mais laisse apparaître quelques différences qu'il convient d'approfondir par une démarche et une lecture plus interprétatives des classes.

\section{La comparaison synchronique}

Il apparaît tout d'abord que les secteurs de l'économie affectés par la crise ne sont pas les mêmes dans les deux pays. Pour l'AFP, il s'agit davantage d'une

3. «Le secrétaire général adjoint de l'organisation du PP, Carlos Floriano, a nié aujourd'hui qu'il existe une démoralisation au sein du parti, face aux perspectives économiques et au chômage, et il s'est montré convaincu que les seules politiques possibles pour faire face à la crise sont celles qu'a lancées le gouvernement». 
économie basée sur les grands secteurs industriels de l'automobile et de l'aviation (classe 1 : Renault, automobile, transport, aérien).

En septembre, mois traditionnellement assez important, les immatriculations de voitures neuves ont baissé de $8,1 \%$ par rapport au même mois de 2009 , selon les chiffres du comité des constructeurs français d'automobiles CCFA publiés vendredi, alors que Paris accueille le mondial de l'auto. (01/10/2010, classe 1 de l'AFP)

En Espagne, même si les secteurs de l'automobile et des compagnies aériennes sont présents (classes 2 et 7 : vehículo, automóvil, aerolinea, vuelo, aéreo), l'EFE cite davantage le secteur agricole (classe 7 : vino, carne, aceite).

La caída de renta que sufrieron los productores durante el ejercicio de 2009, afectó especialmente a los trabajadores del cereal 30 por ciento, aceite de olivas 24 por ciento, leche 21 por ciento y frutas 12 por ciento.4 (26/07/2010, classe 7 de l'EFE)

Mais d'autres résultats marquent une différence plus importante que ces domaines d'activité propres à chacun des pays. Le premier concerne le terme même de "crise économique » : s’il est bien corrélé à la finance dans le corpus français (classe 3 : mondial, économie, récession, pays émergents), et plutôt dans un co(n)texte mondialisé, il n'est pas lié au même champ lexical dans le corpus espagnol, où on le retrouve plutôt avec un vocabulaire plus général et plus socio-politique (classe 4 : problema, político, España).

Il est plus intéressant encore de noter que cette classe 4 de l'EFE partage la même classe-mère que les considérations sur les réformes engendrées par la crise (classe 3)5. Le terme même de «crise économique » n'est donc pas lexicalisé de la même façon en France et en Espagne : il est davantage lié aux décisions politiques et nationales pour la presse espagnole, à la finance mondialisée pour la presse française. C'est la raison pour laquelle on trouve, dans l'analyse de l'EFE, une classe dédiée aux mesures d'austérité qu'ont adoptées les deux gouvernements espagnols pour sortir de la crise (classe 3), ce qui n'est pas le cas dans l'analyse du corpus AFP.

La vicepresidenta primera del gobierno, María Teresa Fernández de la Vega, confió en que la austeridad de los presupuestos de 2011, junto con la reforma laboral acometida, el proyecto de ley de economía sostenible y la futura reforma de las pensiones, hagan crecer el empleo. ${ }^{6}$ (24/o9/2010, classe 3 de l'EFE)

La question de la politique internationale, ensuite, est présente dans les deux corpus, mais il y a une différence dans la manière de l'aborder. Dans le

4. "La baisse des revenus qu'ont subie les producteurs au cours de l'année 2009 a touché particulièrement les producteurs de céréales, 30\%, d'huile d'olive $24 \%$, de lait $21 \%$ et des fruits $12 \%$ ».

5. C'est-à-dire que ces deux classes sont issues de la même branche.

6. «La vice-Première ministre, Maria Teresa Fernandez de la Vega, espère que l'austérité dans le budget de 2011, en même temps que la réforme du travail, le projet de loi sur l'économie durable et la future réforme des retraites, fera augmenter l'emploi ». 
corpus espagnol, la politique internationale investit deux lexiques. Un lien est établi entre Europe et crise (classe 6 : Union Europea, Bruselas, Barroso) ; ce n'est pas étonnant pour un pays qui, ayant obtenu des aides économiques du Fonds monétaire international (FMI) et de la Banque centrale européenne (BCE), doit intégrer les obligations que ces organismes lui imposent et qui vont marquer la politique nationale du pays. Un autre lexique lié à l'international (classe 9) évoque, lui, les États-Unis (Obama, Estados Unidos, Casa Blanca) comme origine de la crise et puissance économique qui s'impose au reste du monde, mais également l'Amérique du Sud (Colombia, Argentina), pour ses relations historiques, culturelles et économiques avec l'Espagne.

Dans le corpus français, la crise est davantage traitée comme un problème mondial et l'Europe est présentée comme l'instance chargée d'y faire face et éventuellement comme ayant une part de responsabilité dans l'arrivée de cette crise (loi, réformes, Commission européenne). Les États-Unis sont mentionnés également, mais dans une moindre mesure et dans un contexte concernant aussi d'autres pays et acteurs internationaux qui impliquent d'autres crises (Russie, Iran, etc.).

La croissance mondiale durablement en panne, malgré les pays émergents. La crise économique et financière va durablement peser sur la croissance mondiale, la demande intérieure des pays émergents restant trop faible pour compenser la récession des pays développés. (04/06/2009, classe 3 de l'AFP)

Une thématique importante dans les deux classifications renvoie à la question politique. Mais, ici encore, la politique n'est pas traitée de la même façon par les deux agences de presse. L'agence espagnole développe un point de vue plus institutionnel, donnant plus d'importance aux partis politiques (classe 8 : partido, PP, PSOE, socialista) et aux syndicats (classe 5 : UGT, CCOO, sindicato). La politique espagnole est évoquée directement en lien avec les problématiques économiques, la responsabilité vis-à-vis des réformes, l’État espagnol, les syndicats et l'Europe.

El PP es según Gallardón un partido firme en sus principios y convicciones, un partido que aunque esté ahora en la oposición es un partido de gobierno, con un liderazgo reforzado que encarna la figura de Mariano Rajoy elegido por todos sus compañeros y con un equipo renovado.7 (28/06/2008, classe 8 de l'EFE)

Los secretarios generales de CCOO Ignacio Fernández Toxo y UGT Cándido Méndez encabezan esta movilización que tiene por lema, por el empleo con derechos y la garantía de nuestras pensiones. ${ }^{8}$ (01/05/2010, classe 5 de l'EFE)

7. «Le PP est, selon Gallardón, un parti solide dans ses principes et ses convictions, un parti qui, même s'il est maintenant dans l'opposition, est un parti de gouvernement, avec un renforcement du leadership incarné par la figure de Mariano Rajoy, élu par tous ses camarades et avec une équipe renouvelée».

8. «Les secrétaires généraux du CCOO (Commissions Ouvrières), Ignacio Fernández Toxo, et de 
Du côté français, au contraire, la politique et la crise économique apparaissent dans des espaces lexicaux très distincts, comme si les deux n'avaient pas de rapport. Il y a quatre classes liées au politique (classes 4, 5, 6 et 8). On y observe une tendance à la personnalisation (classe 4) mettant en scène des personnalités placées en confrontation (Hollande, Sarkozy, Aubry, Fillon, etc.). Damon Mayaffre (2003) montrait, dans son étude des corpus politiques contrastifs en France, qu'il existe des individualisations linguistiques qui correspondent aux identités politiques des orateurs. Le discours politique de la crise est effectivement ici affaire d'identité, puisqu'il construit un espace linguistique de reconnaissance pour le public. Et cela s'accompagne d'une forte référence aux sondages d'opinion (classe 6) et à la projection de toute information dans un calendrier électoral. Tout semble se passer comme si, pour la presse française, quel que soit le moment et quel que soit l'objet, toute personnalité politique était immédiatement traitée comme un ou une candidate potentielle dans une échéance électorale plus ou moins lointaine qu'il s'agit d'anticiper. Ce mode de traitement extrêmement personnalisé se retrouve également au niveau international (Obama, Bush, Mohamed, etc.) et une partie de la politique internationale est volontiers abordée au travers du prisme des rencontres avec nos propres dirigeants (classes 5 et 8 ).

\section{La dynamique diachronique}

Les analyses précédentes ont permis d'identifier des différences sur le lexique mobilisé par les agences française et espagnole à propos de la crise économique et sur une période de six ans.

Mais si la crise a connu des événements d'importance mondiale (krach de la bourse nord-américaine, sauvetage de l'économie grecque avec des aides européennes), elle se traduit également, pour chaque pays, par des événements nationaux, qu'il s'agisse d'évaluations par les agences internationales de notation, d'échéances électorales, de décisions touchant la vie quotidienne des citoyens, etc.

La période analysée (2008-2014) est marquée, autant en France qu'en Espagne, par un contexte social et politique perturbé impliquant des mesures contraignantes. Étudier les représentations sociales sous l'angle chronologique peut donc permettre d'en saisir les constantes et les évolutions lexicales. Il s’agit ici de voir si les périodes sont associées à des cadrages thématiques particuliers.

Il est possible de projeter les classes lexicales définies par la CDH sur la chronologie des articles (en utilisant le mois comme unité : voir Ratinaud, 2014 ;

I’UGT (Union Générale des Travailleurs), Cándido Méndez, sont en tête de cette mobilisation, qui a comme slogan, pour un emploi avec des droits et la garantie de nos retraites ». 
Ratinaud et Marchand, 2015) et les graphiques 5 (AFP) et 6 (EFE) rendent effectivement compte de la sur-apparition de quelques thématiques à certains moments.

Sur ces graphiques (page suivante), la largeur des colonnes est proportionnelle à l'intensité des informations (mesurée par le nombre de segments de texte pour une période donnée), la hauteur des lignes est proportionnelle à la taille des classes et les cases sont coloriées lorsque la surreprésentation d'une date est significative (au sens du $\chi^{2}$ ) pour la classe concernée. Ces graphiques permettent donc de suivre précisément l'évolution chronologique des thématiques.

Sur les deux graphiques, on voit clairement que les différentes thématiques ne sont pas traitées de façon constante dans la période considérée, mais qu'elles définissent des moments-clés de la situation historique de chaque pays.

Dans le corpus AFP, le début de la période analysée surinvestit les thématiques économiques (classes 1, 2, 3 et 7), au travers des questions financières, monétaires et des secteurs de l'activité qui commencent à être touchés. La politique internationale et l'Europe sont surreprésentées en 2008 (classes 5 et 8), au moment du déclenchement de la crise, mais leur proportion tend à diminuer avec le temps. D'autres thématiques prennent alors plus d'importance. Plus précisément, on observe une rupture thématique en juin 2011, moment auquel l'AFP commence à moins évoquer la macroéconomie, le budget et la mondialisation (classes 1, 2, 3 et 7) pour investir les aspects nationaux, électoraux et culturels (classes 4, 6 et 9).

Le même phénomène apparaît, et de façon plus remarquable encore, pour l'agence EFE, où le niveau économique (classes 1, 2 et 7) et l'Europe (classe 6) dominent dans un premier temps, pour changer brutalement à la fin 2011, et laisser la place à des questions liées aux mesures d'austérité (classe 4), et à des aspects plus sociaux qui impliquent la politique nationale (classes 8 et 9).

Les deux agences passent donc d'un cadrage économique à un cadrage politique, entre 2010 et 2011 pour la France, entre 2011 et 2012 pour l'Espagne. Plusieurs facteurs explicatifs de ces ruptures nous semblent pouvoir être avancés, au premier rang desquels le chômage, le changement de gouvernement et les affaires politico-financières.

\section{Le chômage}

Même si la crise économique a touché plusieurs aspects de l'économie espagnole, la dégradation de l'emploi a été l'une des conséquences les plus évidentes et les plus graves de cette récession économique, puisque le nombre de chômeurs a dépassé les six millions au début 2013. Le mot desempleo (chômage) est présent dans trois classes de notre classification (classes 1, 4 et 5), 
Le traitement de la crise économique par les agences de presse

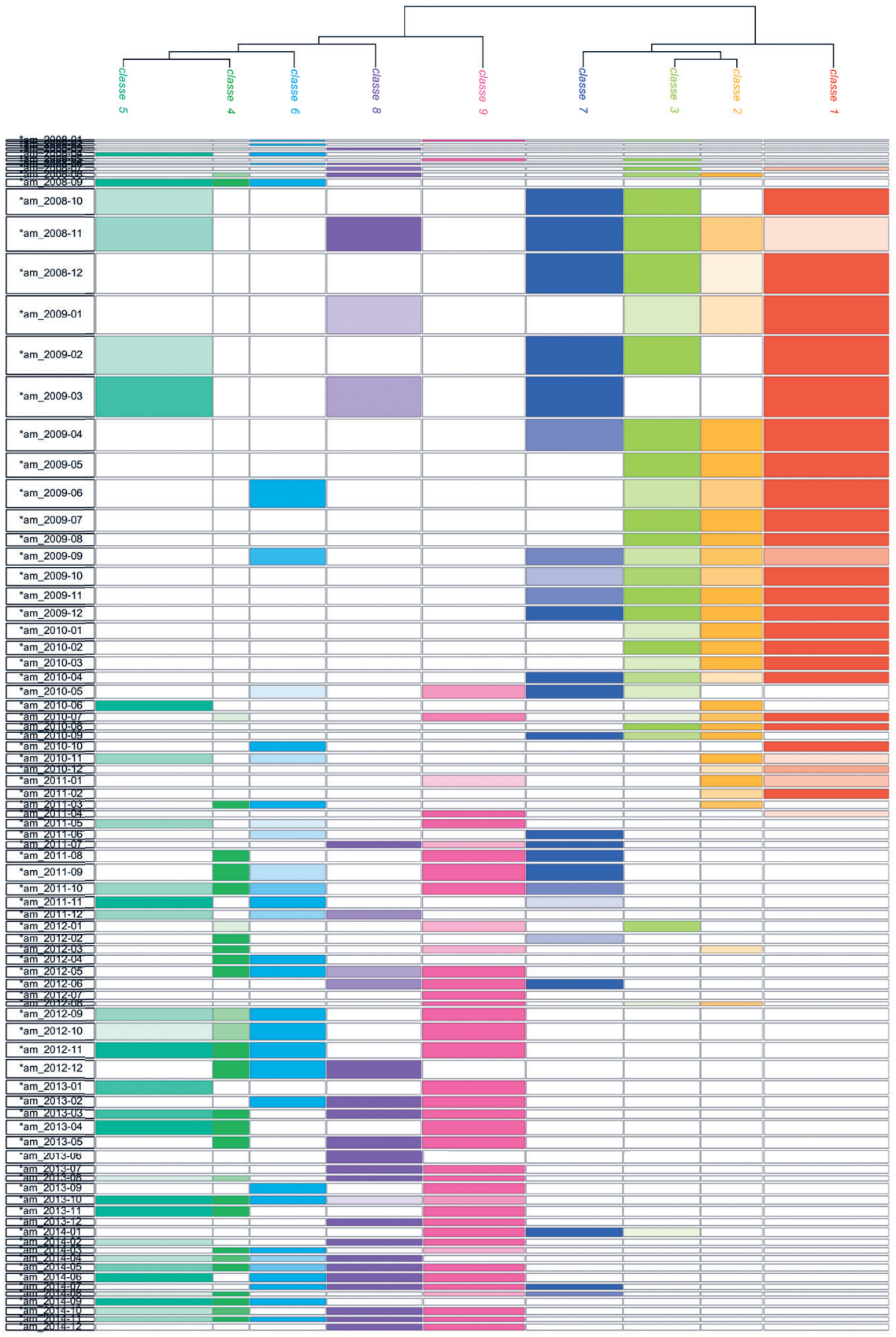

Figure 5 : Dendrogramme de la CDH et représentation chronologique des classes du corpus «AFP»

Mots. Les langages du politique $\mathrm{n}^{\circ} 115$ novembre $2017 \bullet 65$ 

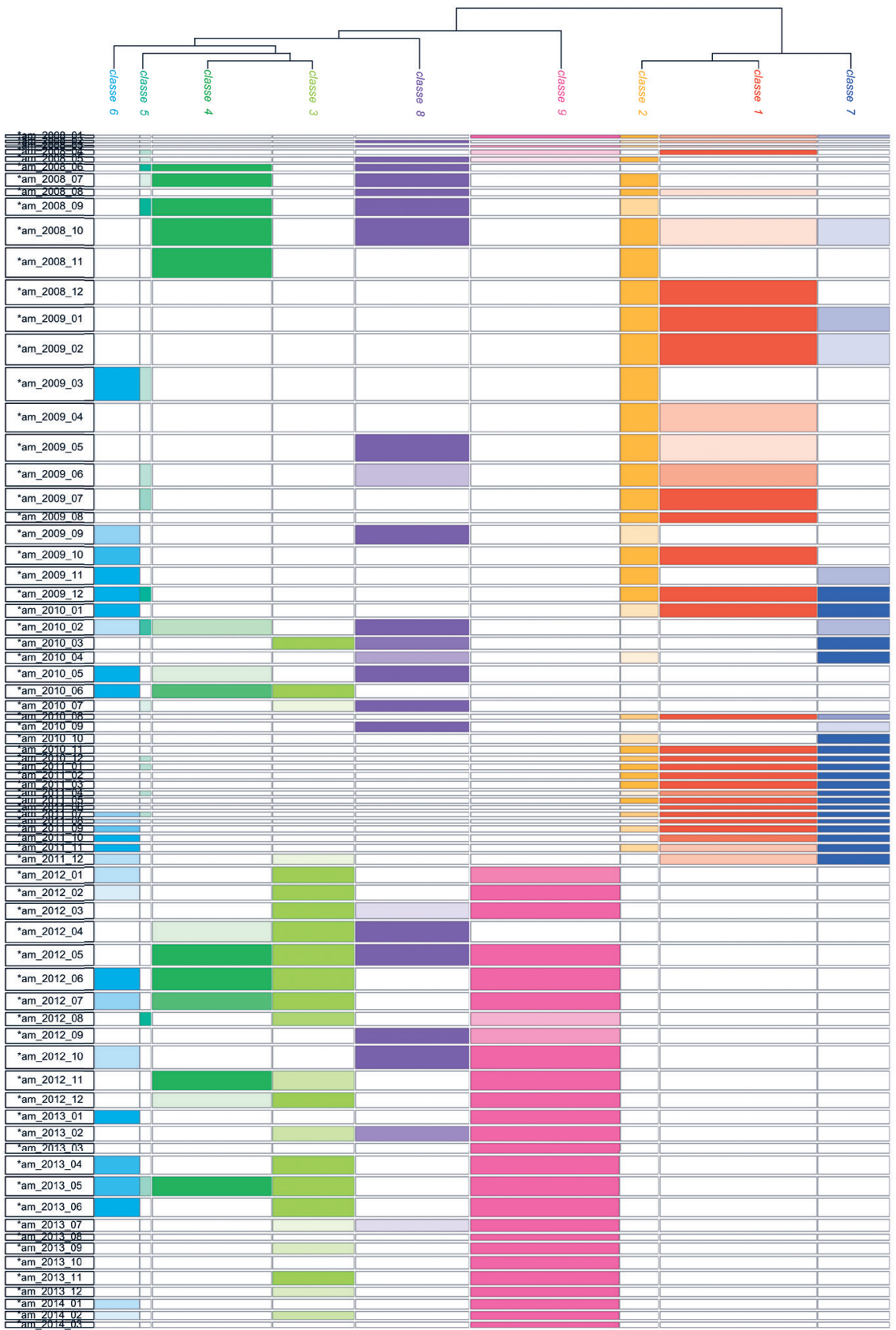

Figure 6 : Dendrogramme de la CDH et représentation chronologique des classes du corpus «EFE» 
mais de la façon la plus significative dans la classe $1\left(\chi^{2}: 3425,526\right)$, où il fait référence à un indicateur économique mesurant l'état du pays. Cette thématique va être traitée par l'EFE surtout de 2008 à 2011.

Con la crisis económica en 2009 el país sufre una recesión del 5,8 por ciento. La tasa de desempleo se situó en un 14,8 por ciento en 2009, pero se pronostica que debido a la recesión podría alcanzar en 2010 un 15, 6 por ciento. 9 (23/12/2009, classe 1 de l'EFE)

Desempleo apparaît également, mais moins massivement, dans les classes $4\left(\chi^{2}: 61,457\right)$ et $5\left(\chi^{2} 17,759\right)$, à partir de 2012 , mais il est contextualisé différemment, avec les politiques d'austérité ou en référence à la situation de l'emploi (droits, licenciements, etc.) et dans le cadre de négociations entre les syndicats et le gouvernement.

Ante la situación del país, la que González ha conminado al presidente del gobierno Mariano Rajoy a que cambie radicalmente sus políticas, y a que reconozca que después de un año de vigencia la reforma laboral ha generado más desempleo. ${ }^{10}$ (04/03/2013, classe 4 de l'EFE)

Toxo (UGT) y Méndez (CCOO) han coincidido en asegurar que no perciben que la sociedad cuestione a los sindicatos, y que por el contrario la convocatoria de huelga y las movilizaciones serán un éxito y obligarán al gobierno a rectificar sobre la reforma laboral. ${ }^{11}$ (24/og/2010, classe 5 de l'EFE)

Dans le corpus de l'agence française, le mot "chômage » est présent dans les classes 2 et 3. Comme pour son homologue espagnol, il est abordé comme un indicateur de santé économique du pays, et également en début de période (2009-2010), mais il est davantage contextualisé comme un problème international, en référence à d'autres pays particulièrement frappés par la crise.

Le taux de chômage espagnol atteint lui aussi un niveau record avec 17,36 \% au premier trimestre, ce qui est le plus haut niveau enregistré en Espagne depuis fin 1998, et s'affiche comme un niveau record parmi les pays de la zone euro. (24/04/2009, classe 2 de l'AFP)

Contrairement à ce qui se produit en Espagne, le chômage n'est pas significativement recontextualisé par l'AFP dans un lexique politique en fin de période.

9. «Avec la crise économique, en 2009, le pays a connu une récession de 5,8\%. Le taux de chômage a atteint 14,8\% en 2009, mais il est prévu qu'à cause de la récession, il puisse atteindre $15,6 \%$ en $2010 »$.

10. «Compte tenu de la situation, Gonzalez a exhorté le président du gouvernement, Mariano Rajoy, à changer radicalement ses politiques, et qu'il reconnaisse, un an après l'entrée en vigueur de la réforme du travail, qu'elle a généré plus de chômage ».

11. «Toxo (UGT) et Mendez (CCOO) pensent que les citoyens n'ont pas perdu confiance en eux, et qu'au contraire, l'appel à la grève et aux manifestations seront un succès. Ils prévoient que cette manifestation va faire reculer le gouvernement et rectifier la nouvelle réforme du travail ». 


\section{Les changements politiques}

Les deux pays sont marqués par des changements politiques importants sur la période 2008-2014.

En France, le début de la période étudiée coïncide avec l'arrivée d'un nouvel exécutif (Nicolas Sarkozy, président de la République ; François Fillon, Premier ministre), confronté à la récession et attendu sur les mesures pour y faire face. Mais il s'agit également d'un contexte marqué par un certain nombre de troubles politiques internes : une série de polémiques ont brouillé les valeurs traditionnelles de la droite au pouvoir, la politique sécuritaire se durcit avec la création du ministère de l'Immigration, de l'Intégration, de l'Identité nationale et du Développement solidaire, des réformes sociales (notamment sur les retraites) divisent l'opinion. En juin 2011, la campagne de l'élection présidentielle de 2012 démarre avec les candidatures à la primaire citoyenne organisée par le Parti socialiste (PS). Or, cette période correspond justement au changement de cadrage thématique dans les dépêches de l'AFP. En 2012, l'élection présidentielle amène un changement d'exécutif avec l'élection de François Hollande (PS) et la nomination de Jean-Marc Ayrault comme Premier ministre, remplacé deux ans plus tard par Manuel Valls. Ce nouveau gouvernement aura aussi la tâche d'essayer de freiner les déséquilibres et la dégradation des indicateurs économiques : contraction économique, absence de croissance, augmentation du taux du chômage. Contrairement à ce que l'on aurait pu attendre, on n'observe pourtant pas de retour significatif des thématiques économiques dans les dépêches de l'AFP. Il y a davantage un traitement de la politique nationale/internationale et des affaires sociales.

En Espagne, le début de la période étudiée coïncide avec la deuxième législature de José Luis Rodríguez Zapatero - Partido Socialista Español (PSOE), parti de gauche. Il conduit un gouvernement qui doit faire face à l'apparition de la crise économique en prenant des mesures pour essayer de freiner la forte détérioration des indicateurs économiques, comme la hausse du chômage ou la baisse du PIB. Des décisions radicales sont prises (baisse de $5 \%$ du salaire des fonctionnaires, etc.). Septembre 2010 marque la première réforme du travail, mise en place par le gouvernement de Zapatero (PSOE), qui donne lieu à une grève générale : les syndicats vont utiliser la question du chômage comme une preuve de la mauvaise gestion du gouvernement. En novembre 2011, M. Zapatero convoque des élections anticipées et Mariano Rajoy - Partido Popular (PP), parti de droite - le remplace à la Moncloa (palais présidentiel du gouvernement espagnol) en décembre 2011. Il adopte une série de mesures d'austérité drastiques pour pallier une situation jugée comme étant «dans le rouge» (hausse de la TVA de $18 \%$ à $21 \%$; augmentation de $20 \%$ des effectifs d'élèves par classe dans l'enseignement public...). Ici encore, on n'assiste 
pas à un retour des thématiques économiques après la mise en place du nouveau gouvernement.

\section{Les affaires politico-financières}

En Espagne comme en France, la période 2008-2014 est marquée par des délits financiers impliquant des personnalités politiques. Mais seul le corpus espagnol comporte des références à ces affaires, et plus précisément à la « corruption» (essentiellement classe 8), tant à droite (PP), avec les affaires Gürtel, Bárcenas et Nóos, qu'à gauche (PSOE) avec le cas des ERE de l'Andalousie ${ }^{12}$. À cela s'ajoute la tension nationaliste de Catalogne à laquelle les deux gouvernements tentent de faire face. Ces références ne suivent pas la même chronologie que les thèmes précédents et le traitement du thème de la corruption semble suivre le cours de la découverte et du traitement de ces affaires. En revanche, la présence de ce vocabulaire dans notre corpus révèle qu'il est mis en contexte avec la crise économique.

En France, au contraire, des affaires de fraude ou d'évasion fiscales existent et sont traitées par l'AFP, mais ne sont pas contextualisées avec la crise économique.

\section{Discussion}

À partir d'un corpus portant sur la crise économique telle qu'elle est traitée par les deux principales agences de presse française et espagnole, nous pouvions poser deux hypothèses : soit des traitements analogues renverraient à des thématiques communes et à des approches similaires de ce phénomène mondial, soit des différences de traitement amèneraient à penser que l'information est abordée de façon spécifique par chaque pays.

Une première analyse (analyse de similitude) a permis de visualiser la macrostructure de chaque corpus (distribution générale des formes pleines de haute fréquence) et de montrer une convergence entre les deux corpus. On peut alors être tenté de conclure à un traitement généralisé de l’information médiatique, reposant notamment sur des représentations professionnelles, acquises et partagées par les journalistes pour asseoir, non seulement leurs pratiques, mais également leur identité professionnelle (Bataille etal., 1997). Ce sont ces «routines» journalistiques, issues de formations et parcours professionnels

12. «ERE : Expediente de regulación de empleo (Procédure de licenciement économique)». Entre 2001 et 2010, le gouvernement régional andalou (présidé par le PSOE) a approuvé de fausses préretraites dans le contexte des ERE. L'administration a comptabilisé jusqu'à 235 bénéficiaires considérés comme suspects. Par ailleurs, 887 subventions irrégulières ont été octroyées à des entreprises (ces aides ont été gérées par le directeur général de l'emploi de cette région). 
de plus en plus partagés sur le plan international, qui seraient susceptibles d'homogénéiser le contenu des informations pertinentes pour une industrie médiatique mondialisée.

Mais des analyses supplémentaires (classifications lexicales automatiques) mettent en évidence des microstructures, qui distinguent des traitements spécifiques par les agences française et espagnole et révèlent des choix ou des agencements thématiques propres à chaque pays. Au-delà des grands cadres communs, la contextualisation de la crise n'est donc pas la même dans les deux agences. Par exemple, là où l'agence française établit des rapprochements avec la finance mondiale et les relations internationales, son homologue espagnole intègre des problématiques nationales aux conséquences internes (perte de confiance dans les acteurs politiques, corruption, spéculation immobilière et bancaire...).

Au-delà de cette co-textualisation synchronique de la crise, la dynamique chronologique a permis de mettre en évidence la succession des thématiques et l'importance d'un événement politique dans l'organisation du discours médiatique. Ainsi donc, dans les deux agences, on observe une rupture thématique coïncidant avec une élection. Le changement dans l'agenda politique engendré par le début d'une campagne électorale ou le changement d'un gouvernement peut logiquement correspondre à une modification des thématiques priorisées par les journalistes. Dans le cas de l'EFE, ce phénomène est potentiellement amplifié par le changement du président de l'agence qui intervient alors par décision du Parlement.

On observe néanmoins un résultat inattendu : pour les deux agences française et espagnole, une fois l'élection passée et le gouvernement mis en place, il n'y a pas de retour significatif du lexique économique, puisque la politique nationale et les questions plus sociales restent davantage traitées.

L'approche textométrique permet donc de reconstruire l'histoire à partir des relations existantes entre textes (productions médiatiques), co-textes (structures lexicales formalisées) et contextes (événements socio-historiques). Cela nous ramène à la problématique de l'ancrage, qui permet de rendre compte de l'insertion des représentations sociales dans le système des croyances générales (Doise, Clémence et Lorenzi-Cioldi, 1992, p. 264). Le discours médiatique se construit sous l'influence conjuguée des représentations professionnelles des journalistes, qui homogénéisent le traitement de l'information, et des représentations sociales partagées au sein d'un collectif national, qui ancrent la crise économique dans des savoirs signifiants pour la population cible. 


\section{Références}

BATAILle Michel, Blin Jean-François, JACQUet-Mias Christine, PIASER Alain, 1997, « Représentations sociales, représentations professionnelles, système des activités professionnelles ", L'Année de la recherche en sciences de l'éducation, Paris, PUF, p.57-89.

Bourdieu Pierre, 1972, Esquisse d'une théorie de la pratique, Genève, Droz.

BURGUET Annette, 2000, "Le contrat de communication dans le discours de la presse », Revue internationale de psychologie sociale, $\mathrm{n}^{0}{ }^{13}$, p. 151-180.

CAStERAn Claude, 2012, Aux sources de l'info: Agence France-Presse, Arles, Actes Sud junior.

De Rosa Annamaria Silvana, Boccı Elena et BulgarelLa Clio, 2010, «Économie et Finance durant la crise financière mondiale : représentations sociales, métaphores et figures rhétoriques dans le discours des médias de l'automne 2008 au printemps 2010 ", Les Cahiers internationaux de psychologie sociale, n87, p. 543-584.

DoIsE Willem, 1982, L'Explication en psychologie sociale, Paris, PUF.

DoIse Willem, CLÉMENCE Alain et LORENZI-CIOLDI Fabio, 1992, Représentations sociales et analyses de données, Grenoble, PUG.

GHIGlione Rodolphe et CHABRol Claude, 2000, «Contrats de communication, stratégies et enjeux», Revue internationale de psychologie sociale, nº 4, p. 7-15.

JODELET Denise, 2011, «Dynamiques sociales et formes de la peur», Nouvelle revue de psychosociologie, $\mathrm{n}^{0} 12$, p. 239-256.

LAGNEAU Éric, 2003, «Agencier à l'AFP : l'éthique du métier menacée», Hermès, La Revue n०35, p. 109-118.

Marchand Pascal et Ratinaud Pierre, 2012a, Être français aujourd'hui. Les mots $d u$ "grand débat» sur l'identité nationale, Paris, Les liens qui libèrent.

-, 2012b, «L'analyse de similitude appliquée aux corpus textuels : les primaires socialistes pour l'élection présidentielle française (septembre-octobre 2011) », Actes des ${ }_{11}$ e Journées internationales d'Analyse statistique des données textuelles (JADT), p. 687-699.

MAYAFFre Damon, 2003, "Dire son identité politique», Cahiers de la Méditerranée, no66, p. 247-264.

Moscovici Serge, 1984, "The Phenomenon of Social Representations», Social Representations, R. Farr et S. Moscovici, Paris, Éd. de la MSH ; Cambridge, Cambridge University Press p. 109-118.

-, 1961, La Psychanalyse, son image et son public, Paris, PUF.

PALmer Michael, 2009, «Transnationales de l'information », Questions de communication, nº 15, p. 345-366.

RATINAUd Pierre, 2014, "Visualisation chronologique des analyses ALCESTE : application à Twitter avec l'exemple du hashtag \#mariagepourtous », Actes des $12^{e}$ Journées internationales d'Analyse statistique des données textuelles, Paris, p. 553-565.

RATINAUd Pierre et MARChAND Pascal, 2015, «Des mondes lexicaux aux représentations sociales. Une première approche des thématiques dans les débats à l'Assemblée nationale (1998-2014) », Mots. Les langages du politique, $\mathrm{n}^{0}{ }^{108}$, p. 57-77.

-, 2012, «Application de la méthode ALCESTE à de "gros" corpus et stabilité des “mondes lexicaux" : analyse du "CableGate" avec IRaMuTeQ», Actes des $11^{e}$ Journées internationales d'Analyse statistique des données textuelles, p. 835-844. 
REINERT Max, 1983, «Une méthode de classification descendante hiérarchique : application à l'analyse lexicale par contexte », Les Cahiers de l'analyse des données, nº3, p. 187-198.

REINERT Max, 1990, «ALCESTE : une méthodologie d'analyse des données textuelles et une application : Aurélia de Gérard de Nerval», Bulletin de méthodologie sociologique, $\mathrm{n}^{0} 26$, p. 24-54.

Rizzolı Valentina, Romaioli Diego et ConTARello Alberta, 2017, «The Crisis Tsunami. Social Representations of the Economic Crisis in the Italian Press», International Review of Social Psychology, ${ }^{\circ}{ }^{\circ}$ o, p. 80-91.

RouQUETTE Michel-Louis, 1999, «Sur une catégorie particulière de représentations sociales en psychologie politique », Psychologie \& société, n² 2, p. 33-41.

-, 1994, Sur la connaissance des masses. Essai de psychologie politique, Grenoble, PUG.

-, 1988, La Psychologie politique, Paris, PUF.

VERón Eliseo, 1985, «L'analyse du contrat de lecture : une nouvelle méthode pour les études de positionnement des supports presse », Médias : expériences, recherches actuelles, applications, Paris, Institut de recherches et d'études publicitaires. 